\title{
A placebo-controlled clinical trial to evaluate the effectiveness of massaging on infantile colic using a random-effects joint model
}

This article was published in the following Dove Press journal:

Pediatric Health, Medicine and Therapeutics

\author{
Samaneh Mansouri' \\ Iraj Kazemi² \\ Ahmad Reza Baghestani ${ }^{3}$ \\ Farid Zayeri' \\ Fatemeh $\mathrm{Nahidi}^{4}$ \\ Nafiseh Gazerani ${ }^{4}$ \\ 'Department of Biostatistics, Faculty \\ of Paramedical Sciences, Shahid \\ Beheshti University of Medical \\ Sciences, Tehran, Iran; ${ }^{2}$ Department \\ of Statistics, Faculty of Sciences, \\ University of Isfahan, Isfahan, Iran; \\ ${ }^{3}$ Department of Biostatistics, \\ Faculty of Paramedical Sciences, \\ Physiotherapy Research Center, \\ Shahid Beheshti University of Medical \\ Sciences, Tehran, Iran; ${ }^{4}$ Department of \\ Midwifery and Reproductive Health, \\ Shahid Beheshti University of Medical \\ Sciences, Tehran, Iran
}

Correspondence: Ahmad Reza Baghestani Department of Biostatistics, Faculty of Paramedical Sciences, Physiotherapy Research Center, Shahid Beheshti University of Medical Sciences, Ghods Square, Darband Street, Tehran

|97|6533/3, Iran

Tel +98 912 17/ I548

Email baghestani@sbmu.ac.ir
Background: Infantile colic viewed as a non-dangerous prevalent issue could lead to stress in parents and long-term negative consequences in ex-colicky children. Researchers have not been successful in finding a certain treatment for colic symptoms. Studies suggest completely different approaches as its treatment. Massage therapy as an alternative method in reducing colic symptoms has been recommended in several studies.

Methods: A total of 100 colicky infants in a single blind study were randomly specified to two equal groups of intervention and control. Infants in the intervention group received massage for 15-20 minutes once during the day and once at night before sleep, while infants in the control group were rocked for 15-25 minutes when the symptoms of colic appeared. Parents recorded the details of the colic symptoms in a diary every day. All these outcomes were modeled simultaneously via a random-effects joint model.

Results: Among 100 infants included in the analysis, $48 \%$ were female; $91 \%$ of all infants were breastfed and $54 \%$ of them were born via normal vaginal delivery. In general, the effect of massage therapy on colic symptoms was assessed using the joint model. Our findings illustrated that massaging colicky infants would substantially reduce colic symptoms and increase the sleep duration in babies compared with the rocking group $(P<0.001)$.

Conclusion: Massage therapy could be considered as an effective method in reducing colic symptoms. Mean of the symptoms dropped significantly in the intervention group compared with that in the rocking group. Our study also represents that a relevant and correct statistical model could result in more reliable findings.

Keywords: infantile colic, massage therapy, clinical trial, random-effects joint model

\section{Introduction}

Depending on various definitions, locations, and difference in study designs, the incidence of infantile colic is from $10 \%$ to $40 \%$ in infants aged $<3$ months. ${ }^{1}$ The incidence rate of this syndrome in Iran is about $20 \% .^{2}$ Based on Wessel's description of the "rule of three," a healthy infant with crying more than 3 hours a day, 3 days a week, and 3 weeks in succession is colicky.

Despite the fact that colicky crying is not dangerous for infants, it would cause depression and anxiety for their mothers. ${ }^{3}$ Moreover, it would cause marital stress in parents and behavioral problems in children. Previous studies reported high prevalence of paternal depression. Between $2 \%$ and $25 \%$ of fathers of these infants would experience depression. ${ }^{4}$ This condition can probably affect mother-child relationship and has significant burden on health care system and parental caring. 
Although commonly viewed as a minor issue, it also imposes considerable cost to health care system. Infantile colic seems to be a risk factor of childhood migraine without aura. ${ }^{5}$ It could pose problems to ex-colicky children's behaviors and leads to less academic achievements compared with their peers. ${ }^{6,7}$ Shaken baby syndrome could also be considered as a consequence of increased infant crying. ${ }^{8}$

Some studies have shown that colic does not have longterm consequences. ${ }^{9}$ Nonetheless, some others have revealed possible long-term adverse effects of colic are sleeping disorders, problems in family relationship, and even psychological disorders in future lives of colicky infants. ${ }^{10,11}$ Having more often temporal tantrum in ex-colicky children has been recognized as another long-term consequences of colic. ${ }^{6}$

Despite easy recognition, its certain treatment is still unknown and all suggested ones have little or no effect or they have unwanted side effects. ${ }^{12}$ Based on these nonscientifically proven causes, various treatments were proposed, ranging from drug therapies to behavioral strategies. Simethicone and dicyclomine hydrochloride are two very famous drugs prescribed for this symptom. Yet, no study has proven the efficiency of these treatments. . $^{13,14}$

Massage therapy as an alternative method to reduce colic symptoms ${ }^{15,16}$ has also positive influence on weight gain ${ }^{17,18}$ and development of immune system in preterm infants. ${ }^{19,20}$ It has been shown that massaging infants could stimulate sensation which generally leads to pacifying the infants. It can also improve mother-infant relationship. ${ }^{21}$

In reviewing literatures, we could not find any published papers regarding the assessment of massage therapy on number and duration of crying as well as sleep duration in colicky infants. The majority of these studies considered duration of crying or infant's sleep duration as their only outcome..$^{15,16}$ However, there are studies in which all these outcomes were considered to determine other methods' effectiveness on infantile colic rather than massage therapy. ${ }^{22}$ For this reason, we decided to conduct a study to assess the effectiveness of massage therapy as an alternative treatment in infants with colic symptoms using all the abovementioned responses as outcomes of our study.

\section{Material and methods}

This was a randomized, single-blind, placebo-controlled trial study to evaluate the effect of massaging as a treatment to pacify infantile colic symptoms. One hundred full-term birth colicky infants aged younger than 12 weeks were randomly allocated to two equal groups (50 infants in each group) of massaging and rocking.
At first stage, the study protocol was registered in the Iranian Registry of Clinical Trials (IRCT), with a registration number of IRCT201106054317N5. Parents of colicky infants were informed about the aim of our study before recruiting. They also signed an informed consent form. To calculate the sample size of this study, the results of similar studies ${ }^{23}$ were considered with a significant level of 0.05 and power of $80 \%$. Based on consultation with pediatricians, one hour was determined as a significant difference between the mean of cry duration in the two groups. Based on this information, the minimum sample size of 47 infants per group was determined. In case of withdrawal, we considered 50 samples in each group.

Infants who were brought to pediatric clinic of Amirkabir hospital of Arak were examined by a pediatrician based on Wessel's criteria. Selected infants were randomly assigned into the massage and rocking groups based on their referral day. Hospital staff, such as pediatrician, were kept blind to the allocation. However, it was impossible to apply blinding method for the family of the infants.

The pediatrician and hospital's staff were unaware of the treatment of each group. However, because of the nature of our treatments, it was impossible to blind the methods for mothers. Using any other treatments, having allergy to lactose, and suffering from any other known diseases were considered as exclusion criteria. Moreover, infants with mothers smoking during pregnancy were also excluded. Among the infants passing these criteria, those without skin problems or other limitations on receiving massage were recruited. Parents were taught to distinguish between colicky crying of their babies with other types of crying. After recruiting the study participants, their parents were trained to how to massage or rock their babies. They were asked to do what they were taught in front of our specialists so as to correct their possible mistakes. This test was performed during and after our study in order to recognize and exclude parents who do the massage or rocking in a wrong way. The infants' parents were asked to massage infants for 15-20 minutes once during a day and once at night before sleeping during one-week period. Mothers in the control group were asked to rock their infants gently for 15-25 minutes when the symptoms of colic appeared. All parents recorded the duration of crying and sleep of their infants (hours per day) as well as the number of colic crying throughout the day for one and two days before starting the intervention, which was considered as baseline information. These outcomes were also recorded in a diary for 1 week after starting the study on a daily basis. A committee including 10 experts in midwifery, obstetrics and gynecology 
pediatrics, and neurophysiology verified the validity of the information diary. To have more valid registration, parents were guided by two members of our research team who were blind to the allocation.

\section{Ethical consideration}

The participants of this study could withdraw any time they wished. Each participant also signed a written informed consent. Our study was conducted in accordance with the Declaration of Helsinki and was approved by the Ethics Committee of Shahid Beheshti University of Medical Sciences. The reference number of ethical approval was 116/1769. Due to ethical considerations, we are not allowed to share individual information or the de-identified participant data.

\section{Statistical analysis}

All statistical analyses were conducted using SAS version 9.4. We used Pearson's chi-squared test and independent samples $t$-test for categorical and continuous variables, respectively, to assess the statistically significant differences in levels of demographic and confounder variables. We also used Mann-Whitney U nonparametric test for non-normal data. Furthermore, since we were confronted with situations in which multiple outcomes were recorded simultaneously and measured repeatedly over time, a statistical method that jointly models these outcomes and considers the relationships among them could be applied. Thus, we applied randomeffects joint model of Poisson and normal distributions to explore the effect of intervention on all three responses at the same time in the presence of other variables and consideration of within-subject association and associations among different responses of each person. $P$-value $<0.05$ was considered statistically significant.

\section{Results}

All infants completed the study because no adverse effects were reported during the trial and none of the parents refused to continue our study. In the present study, as shown in Table 1, among 100 infants included in analysis, 48\% were girls and 52\% were boys. Mean age of infants in intervention and control groups was 4.32 and 4.8 weeks, respectively. Mean age of infants' mothers was about 27 years; $91 \%$ of all infants were breastfed and $54 \%$ of them were born via normal vaginal delivery. Median of gravidity was 2 , which means number of pregnancy in more than half of mothers was 2 . The mean of days from starting colic for all infants was about 10 days. As illustrated in Table 1, no significant differences were found in demographic information between the massage group and the rocking group at the significant level of $5 \%$. All variables with $P$-values $<0.2$ were considered in our final joint modeling process so as to consider their confounding effects on our final model.

Table 2 shows the changes in means of colic symptoms during the study. As can clearly be seen, the number and duration of crying in the massage group had more reduction compared with those in the rocking group. The mean number of crying in the massage group was about six at baseline and it became about four at day 7 of our study, while the average number of crying remained almost unchanged in the rocking group. The duration of crying in the massage group experi-

Table I Baseline characteristics of infants in two groups

\begin{tabular}{|c|c|c|c|c|}
\hline Variables & Massage group & Rocking group & Total & $P$-value \\
\hline Sex & & & & 0.23 \\
\hline Boy & $21(42 \%)^{a}$ & $27(54 \%)$ & $48(48 \%)$ & \\
\hline Girl & 29 (58\%) & $23(46 \%)$ & $52(52 \%)$ & \\
\hline Infant age (weeks) & $4.32 \pm 3.23^{c}$ & $4.8 \pm 3.55$ & $4.56 \pm 3.39$ & 0.481 \\
\hline Mother age (years) & $26.9 \pm 3.87$ & $27 \pm 4.5$ & $26.95 \pm 4.18$ & 0.905 \\
\hline Birth weight (g) & $3229.2 \pm 509.9$ & $3383 \pm 843.51$ & $3306.1 \pm 697.72$ & 0.273 \\
\hline Infant weight (g) & $4|2| .2 \pm 986.03$ & $4 \mid 42.8 \pm 1028.23$ & $4132 \pm 1002.308$ & 0.915 \\
\hline Nutrition & & & & $>0.99$ \\
\hline Breastfeeding & 46 (92\%) & 45 (90\%) & 91 (91\%) & \\
\hline Other & $4(8 \%)$ & $5(10 \%)$ & $9(9 \%)$ & \\
\hline Delivery type & & & & 0.229 \\
\hline Normal vaginal delivery & $24(48 \%)$ & $30(60 \%)$ & $54(54 \%)$ & \\
\hline Cesarean section & $26(52 \%)$ & $20(40 \%)$ & 46 (46\%) & \\
\hline Gravidity & $I(I-3)^{b}$ & $2(I-5)$ & $2(I-5)$ & 0.093 \\
\hline Duration from onset (day) & $8.3 \pm 6.82$ & $|1.22 \pm||.4|$ & $9.76 \pm 9.46$ & 0.123 \\
\hline
\end{tabular}

Notes: ${ }^{\mathrm{a} F r e q u e n c y}$ (percent); ${ }^{\mathrm{b}}$ Median (range); ' Mean $\pm \mathrm{SD}$. 
enced a considerable reduction, from about 5 hours at baseline to more than 1 hour at the last day of our study, whereas in the rocking group, this time reduced by about an hour.

Decline in the duration of sleep as another indicator of colic also increased by close to 4 hours in the massage group. However, this indicator stayed the same over the study period.

Table 3 illustrates the effect of massage or rocking on colic symptoms during the study. As shown in this table, all of our three outcomes were significantly associated with the interaction between time and group variables. It reveals that duration and rate of colicky crying in the massage group reduced considerably during the study $\left(P<0.001, \beta^{2}=-0.401\right.$, -0.056 , respectively). These $P$-values and coefficients indicate that the mean duration of colicky crying of infants in the massage group decreased about half an hour more than the rocking group each day. In addition, the incidence rate of crying reduced about $7 \%$ more $(1-\exp (-0.056-0.016))$ in the massage group than in the rocking group.

Durations and number of colicky crying were considerably lower in final days of the study in the intervention group than in the rocking group which can be inferred by both the negative signs of the coefficients and their values. Furthermore, massage therapy had also positive influence on infants' sleep durations which means sleep time increased substantially during the study in the massage group $(P<0.001$, $\mathrm{g} 2=0.439$ ).

It can also be inferred that time from colic onset has significant effect on sleep time as well as on duration of crying. The longer the baby suffers from colic, the shorter they cry and the more they sleep $(P=0.013, P=0.026$, respectively). It could also decrease the number of daily crying. However, it was not a statistically significant relationship. According to its $P$-value ( $P=0.066)$, this relationship needs more assessment.

Duration and number of colicky cries reduced significantly with increasing gravidity, but sleep duration of colicky babies of mothers with more pregnancy experience was considerably longer ( $P=0.004, P=0.01$, respectively) than babies of mothers with less pregnancy $(P=0.04)$. Although these variables showed significant relationships with colic symptoms, the relationships have been taken into consideration through our model so as to obtain more accurate results regarding our main goal.

Figures 1-3 display the trends of colic symptoms by group from baseline to day 7 . A considerable reduction in the number and duration of crying in massage group was observed, while these symptoms remained approximately stable in the rocking group (Figures 1 and 2). As can be clearly seen in Figure 3, although sleep duration increased substantially in the massage group, it did not change in the rocking group.

\section{Discussion}

Although there is no evidence which proves colicky crying is harmful, it could be a stressor for parents and would impose a dramatic burden to health care system and

Table 2 Trends of colic symptoms means by group during study

\begin{tabular}{|c|c|c|c|c|c|c|c|c|c|}
\hline Symptoms & Group & Baseline & Day I & Day 2 & Day 3 & Day 4 & Day 5 & Day 6 & Day 7 \\
\hline Number of & Massage & $6.12 \pm 1.75$ & $8.34 \pm 1.49$ & $7.6 \pm 1.85$ & $6.46 \pm 1.85$ & $6.02 \pm 2.27$ & $5.32 \pm 2.03$ & $4.82 \pm 1.92$ & $4.26 \pm 1.4$ \\
\hline cries & Rocking & $6.92 \pm 2.91$ & $7.5 \pm 1.83$ & $7.22 \pm 1.53$ & $6.78 \pm I .7 I$ & $6.02 \pm 1.95$ & $6.5 \pm 1.9$ & $6.48 \pm 2.04$ & $6.94 \pm 2.14$ \\
\hline Duration of & Massage & $4.96 \pm 1.37$ & $4.12 \pm 2.08$ & $3.55 \pm 1.97$ & $2.86 \pm 1.5$ & $2 \pm 1.05$ & $1.64 \pm 0.93$ & $1.43 \pm 0.84$ & $1.33 \pm 0.73$ \\
\hline crying (hours) & Rocking & $3 \pm|.3|$ & $2.42 \pm 1.71$ & $2.06 \pm 1.5$ & $2.18 \pm 1.52$ & $1.96 \pm 1.42$ & $2.11 \pm 1.63$ & $2.03 \pm 1.38$ & $2.15 \pm 1.23$ \\
\hline Duration of & Massage & $9.22 \pm 1.76$ & $10.22 \pm 2.92$ & $11.3 \pm 2.89$ & $11.54 \pm 2.57$ & $12.42 \pm 2.17$ & $12.54 \pm 1.83$ & $13.42 \pm 2.26$ & $13.12 \pm 1.9$ \\
\hline sleep (hours) & Rocking & $12.24 \pm 2.98$ & $12.38 \pm 1.74$ & $12.46 \pm 1.42$ & $12.3 \pm 1.85$ & $12.38 \pm 1.72$ & $12.36 \pm 1.64$ & $12.2 \pm 1.64$ & $12.4 \pm 1.55$ \\
\hline
\end{tabular}

Table 3 Results of joint model to assess the effect of massaging on three outcomes related to colic symptoms simultaneously in the presence of other variables

\begin{tabular}{|l|l|l|l|l|l|l|}
\hline & $\begin{array}{l}\text { Duration of } \\
\text { crying }\end{array}$ & $\boldsymbol{P}$-value & $\begin{array}{l}\text { Sleep } \\
\text { duration }\end{array}$ & P-value & $\begin{array}{l}\text { Number of } \\
\text { cries }\end{array}$ & $\boldsymbol{P}$-value \\
\hline$\beta_{\text {days }}$ & $-0.119(0.02)$ & $<0.0001$ & $0.064(0.035)$ & 0.072 & $-0.016(0.007)$ & 0.029 \\
$\beta_{\text {days } \times \text { Intervention }}$ & $-0.401(0.027)$ & $<0.0001$ & $0.439(0.042)$ & $<0.0001$ & $-0.056(0.009)$ & $<.0001$ \\
$\beta_{\text {days onset }}$ & $-0.042(0.016)$ & 0.013 & $0.043(0.019)$ & 0.026 & $-0.004(0.002)$ & 0.066 \\
$\beta_{\text {gravidity }}$ & $-0.514(0.175)$ & 0.004 & $0.527(0.2)$ & 0.01 & $-0.05(0.024)$ & 0.04 \\
\hline
\end{tabular}




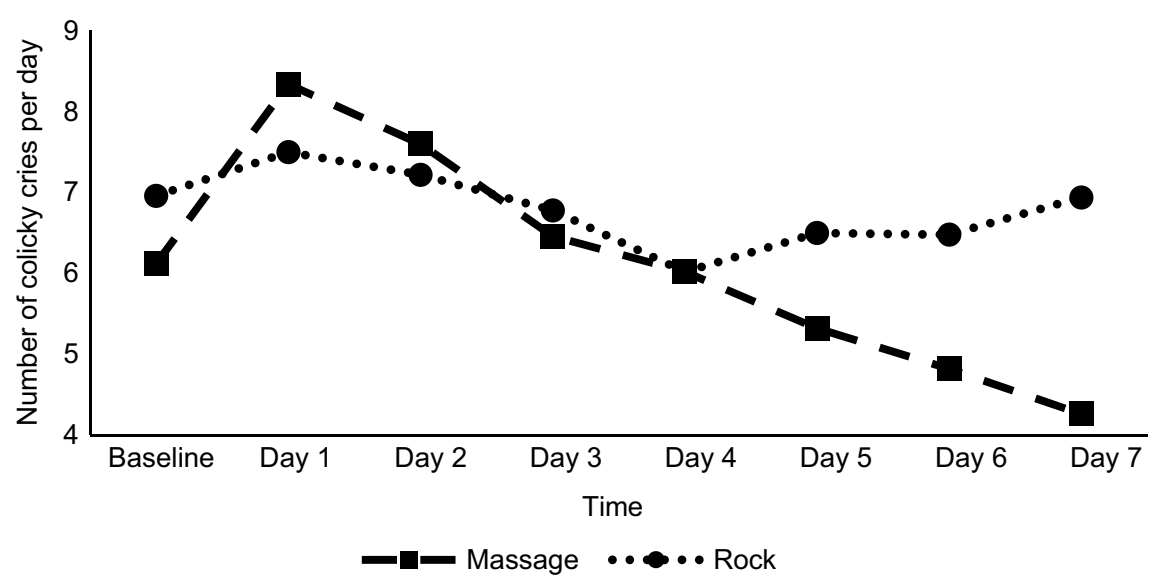

Figure I Trend of number of colicky cries' mean by group.

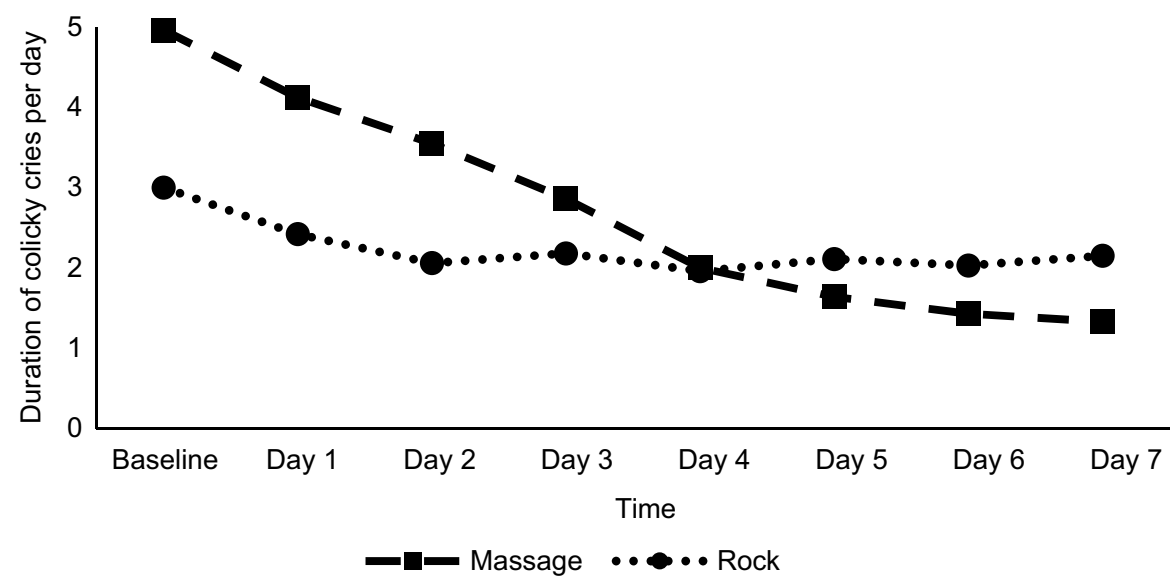

Figure 2 Trends of duration of colicky cries' means by group.

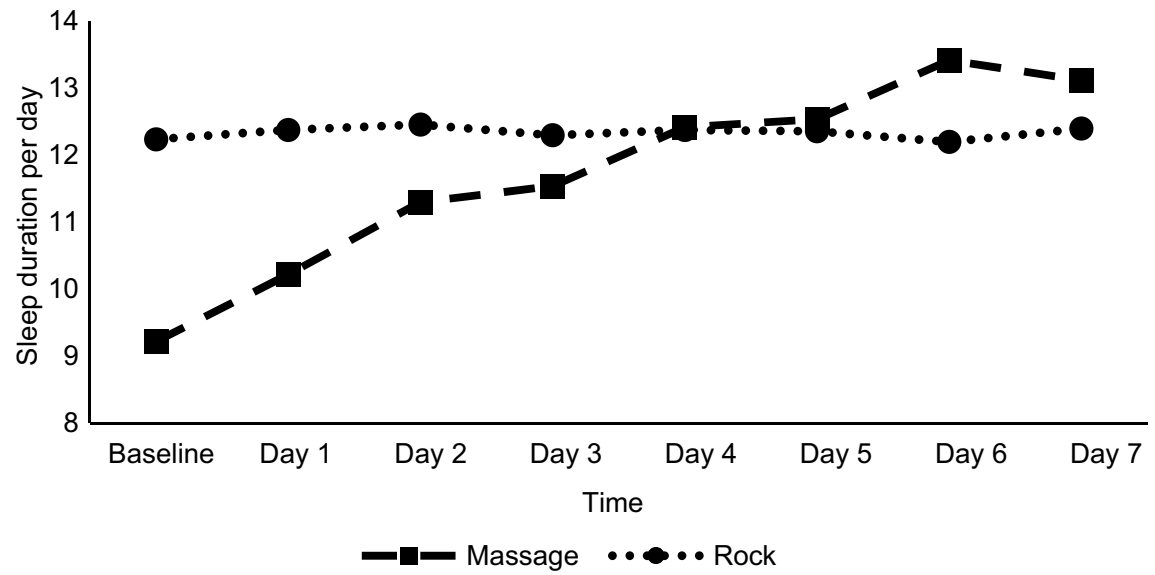

Figure 3 Trends of sleep duration's means by group. 
parental caring. Unfortunately, no proven treatment for curing colic has been introduced. A vast number of studies have suggested various approaches to ease its symptoms, ranging from chemical drugs to traditional medicine. Massage therapy as one of the suggested alternative methods to alleviate these symptoms was applied in this study. We compared the efficacy of massage therapy with rocking on colic symptoms' improvement. Our study revealed that colic symptoms (number and duration of crying) would be mitigated substantially in the massage group. We also illustrated that massage could significantly increase sleep duration.

Although several studies have shown that massage therapy has positive effect on infantile colic syndromes, ${ }^{24}$ none of them have considered correlation of these symptoms, which can be considered only in a joint model. Our study illustrated massage therapy has a positive influence on improving colic symptoms. Since we applied joint model, a sophisticated statistical method which considers all the associations and modeling all responses in a united model, our results are more reliable than those of studies applying separate models. ${ }^{25}$ In joint models, not only the within-subject correlation of such data is taken into account, but association among responses is also considered. Failure to consider these associations may lead to different or even misleading results. ${ }^{26} \mathrm{~A}$ similar study showed that the number of colicky cries could reduce in infants taking massage therapy. ${ }^{27}$

A study conducted to evaluate the effectiveness of massage, sucrose, herbal tea, or hydrolyzed formula in the treatment of infantile colic showed that massage therapy was an effective method in reducing the duration of crying. ${ }^{15}$ However, it was not the most effective method in this study. Another finding revealed that although massage therapy did not reduce gastroesophageal reflux disease symptoms scores, cortisol level indicating stress decreased significantly in infants of the massage group. ${ }^{28}$

A study with the aim of investigating the effect of aromatherapy massage on infantile colic using lavender oil demonstrated that the mean time of weekly crying dropped dramatically in the massage group. ${ }^{16}$ Another study comparing massage with crib vibrator for treating 58 colicky infants $<7$ weeks of age showed the amount of total and colicky crying decreased significantly in both intervention groups. ${ }^{29}$ Although we have not done any investigations on other types of crying, our findings illustrated a significant reduction in colicky crying of infants in the massage group, which is in accordance with the results of previous studies.
As can be seen, numerous studies have suggested massage therapy as a treatment for colic symptoms. ${ }^{30,31}$ However, there are studies that showed that massaging colicky infants is not more effective than placebo. ${ }^{32}$

The majority of studies on infantile colic explored the effect of massage therapy on each of the symptoms separately, while we considered these symptoms as well as sleep duration simultaneously. Findings via applying such sophisticated models would be more reliable. Thus, based on our results, we could infer that massage therapy is an effective factor in reducing colic symptoms compared with rocking colicky infants.

Our study had its own limitations. Since parents performed massage at home, it was impossible to check whether they have massaged correctly. In addition, the similarity of the massages given by different parents and possible mistakes by the parents in calculating sleep and crying duration was also debatable. To overcome the first problem, the performance of parents examined three times: before, during, and after study. Moreover, an advisor was in contact with them to answer their questions during the study. The longitudinal design of our study assisted us to consider each person as their own control. Because our aim was evaluating the colic symptom changes in every participant, the parents only compared with themselves not anybody else, which solves our second problem.

On the other hand, as it was alluded earlier, some studies showed that massage therapy was not a sufficient approach for treating colic, and colic symptoms reduced automatically over time. ${ }^{23}$ These studies lasted for at least 4 weeks which was long enough for curing colic. However, our study was conducted for 7 days. So, it can be inferred that massage therapy decreased colic symptoms and can be considered as an effective treatment for colic.

\section{Disclosure}

The authors report no conflicts of interest in this work.

\section{References}

1. Hall B, Chesters J, Robinson A. Infantile colic: A systematic review of medical and conventional therapies. J Paediatr Child Health. 2012;48(2):128-137.

2. Talachian E, Bidari A, Rezaie MH. Incidence and risk factors for infantile colic in Iranian infants. World J Gastroenterol. 2008;14(29): 4662-4666.

3. McMahon C, Barnett B, Kowalenko N, Tennant C, Don N. Postnatal depression, anxiety and unsettled infant behaviour. Aust NZJ Psychiatry. 2001;35(5):581-588.

4. Smart J, Hiscock H. Early infant crying and sleeping problems: A pilot study of impact on parental well-being and parent-endorsed strategies for management. J Paediatr Child Health. 2007;43(4):284-290.

5. Sillanpää M, Saarinen M. Infantile colic associated with childhood migraine: A prospective cohort study. Cephalalgia. 2015;35(14):1246-1251. 
6. Canivet C, Jakobsson I, Hagander B. Infantile colic. Follow-up at four years of age: still more "emotional." Acta Paediatr. 2007;89(1): 13-17.

7. Rao MR, Brenner RA, Schisterman EF, Vik T, Mills JL. Long term cognitive development in children with prolonged crying. Arch Dis Child. 2004;89(11):989-992.

8. Talvik I, Alexander RC, Talvik T. Shaken baby syndrome and a baby's cry. Acta Paediatr. 2008;97(6):782-785

9. Clifford TJ, Campbell MK, Speechley KN, Gorodzinsky F. Sequelae of infant colic: evidence of transient infant distress and absence of lasting effects on maternal mental health. Arch Pediatr Adolesc Med. 2002;156(12):1183-1188.

10. Savino F, Castagno E, Bretto R, Brondello C, Palumeri E, Oggero R. A prospective 10-year study on children who had severe infantile colic. Acta Paediatr. 2007;94:129-132.

11. Rautava P, Lehtonen L, Helenius H, Sillanpää M. Infantile colic: child and family three years later. Pediatrics. 1995;96(1 Pt1):43-47.

12. Kilgour T, Wade S. Infantile colic. Clin Evid. 2005;13:362-372.

13. Savino F. Focus on infantile colic. Acta Paediatr. 2007;96(9):1259-1264.

14. Lehtonen LA, Rautava PT. Infantile colic: natural history and treatment. Curr Probl Pediatr. 1996;26(3):79-85.

15. Duygu A, Handan A, Gözüm S. Herbal tea or hydrolysed formula in the treatment of infantile colic. J Clin Nurs. 2008;17(13):1754-1761.

16. Çetinkaya B, Başbakkal Z. The effectiveness of aromatherapy massage using lavender oil as a treatment for infantile colic. Int J Nurs Pract. 2012;18(2):164-169.

17. Diego MA, Field T, Hernandez-Reif M. Preterm infant weight gain is increased by massage therapy and exercise via different underlying mechanisms. Early Hum Dev. 2014;90(3):137-140.

18. Diego MA, Field T, Hernandez-Reif M. Vagal activity, gastric motility, and weight gain in massaged preterm neonates. J Pediatr. 2005;147(1): 50-55.

19. Procianoy RS, Mendes EW, Silveira RC. Massage therapy improves neurodevelopment outcome at two years corrected age for very low birth weight infants. Early Hum Dev. 2010;86(1):7-11.

20. Ang JY, Lua JL, Mathur A, et al. A randomized placebo-controlled trial of massage therapy on the immune system of preterm infants. Pediatrics. 2012;130(6):e1549-e1558.
21. White-Traut RC, Nelson MN. Maternally administered tactile, auditory, visual, and vestibular stimulation: relationship to later interactions between mothers and premature infants. Res Nurs Health. 1988;11(1):31-39.

22. Sung V, Hiscock H, Tang ML, et al. Treating infant colic with the probiotic Lactobacillus reuteri: double blind, placebo controlled randomised trial. BMJ. 2014;348:g2107.

23. Huhtala V, Lehtonen L, Heinonen R, Korvenranta H. Infant massage compared with crib vibrator in the treatment of colicky infants. Pediatrics. 2000;105(6):E84.

24. Sheidaei A, Abadi A, Zayeri F, Nahidi F, Gazerani N, Mansouri A. The effectiveness of massage therapy in the treatment of infantile colic symptoms: A randomized controlled trial. Med J Islam Repub Iran. 2016;30:351.

25. Li Q, Pan J, Belcher J. Bayesian inference for joint modelling of longitudinal continuous, binary and ordinal events. Stat Methods Med Res. 2016;25(6):2521-2540.

26. Buhule OD, Wahed AS, Youk AO. Bayesian hierarchical joint modeling of repeatedly measured continuous and ordinal markers of disease severity: Application to Ugandan diabetes data. Stat Med. 2017;36(29):4677-4691.

27. Alvandi SH, Taj Ansari F. Effect of therapeutic touch on colicky pain in infants who admitted in pediatrics privet offices in Tehran. MS thesis in nursing. 1996.

28. Neu M, Pan Z, Workman R, Marcheggiani-Howard C, Furuta G, Laudenslager ML. Benefits of massage therapy for infants with symptoms of gastroesophageal reflux disease. Biol Res Nurs. 2014;16(4):387-397.

29. Huhtala V, Lehtonen L, Heinonen R, Korvenranta H. Infant massage compared with crib vibrator in the treatment of colicky infants. Pediatrics. 2000;105(6):E84.

30. Roberts DM, Ostapchuk M, O’Brien JG. Infantile colic. Am Fam Physician. 2004;70(4):735-740.

31. Hughes S, Bolton J. Is chiropractic an effective treatment in infantile colic? Arch Dis Child. 2002;86(5):382-384.

32. Olafsdottir E, Forshei S, Fluge G, Markestad T. Randomised controlled trial of infantile colic treated with chiropractic spinal manipulation Arch Dis Child. 2001;84(2):138-141.
Pediatric Health, Medicine and Therapeutics

\section{Publish your work in this journal}

Pediatric Health, Medicine and Therapeutics is an international, peerreviewed, open access journal publishing original research, reports, editorials, reviews and commentaries. All aspects of health maintenance, preventative measures and disease treatment interventions are addressed within the journal. Practitioners from all disciplines are invited to submit

\section{Dovepress}

their work as well as healthcare researchers and patient support groups. The manuscript management system is completely online and includes a very quick and fair peer-review system. Visit http://www.dovepress.com/ testimonials.php to read real quotes from published authors. 